\begin{tabular}{|c|c|}
\hline Title & Teraheltz Response of Schottky Wrap Gate Controlled Quantum Dots \\
\hline Author(s) & Kasai, Seiy a; Han, Weihua; Y umoto, Miki; Hasegawa, Hideki \\
\hline Citation & $\begin{array}{l}\text { Physica Status Solidi. C. Current Topics in Solid State Physics, O(4), 1329-1332 } \\
\text { https://doi.org/10.1002/pssc.200303083 }\end{array}$ \\
\hline Issue Date & $2003-06-26$ \\
\hline Doc URL & http:/hdl. handle.net/2115/8457 \\
\hline Rights & $\begin{array}{l}\text { Published in "Physica Status Solidi. C. Current Topics in Solid State Physics", volume 0, issue 4, } 1329-1332 \text { (26 Jun } \\
\text { 2003). }\end{array}$ \\
\hline Type & article (author version) \\
\hline File Information & QD02_G29.pdf \\
\hline
\end{tabular}

Instructions for use 


\title{
Teraheltz Response of Schottky Wrap Gate-Controlled Quantum Dots
}

\author{
Seiya Kasai*, Weihua Han, Miki Yumoto and Hideki Hasegawa
}

Research Center for Integrated Quantum Electronics and Graduate School of Electronics and Information Engineering, Hokkaido University, N13, W8, Kita-ku, Sapporo, 060-8628 Japan

Received 1 October 2002, revised 6 November 2002, accepted

2002

Published online

PACS 73.63.Kv

$\mathrm{THz}$ responses of Schottky wrap gate (WPG)-controlled quantum dots were investigated. Normal incidence $\mathrm{THz}$ irradiation on single-dot and multi-dot devices with a $\mathrm{CH}_{3} \mathrm{OH}$ laser $(2.54 \mathrm{THz})$ changed conductance behavior and produced an additional conductance peak in the $I-V$ characteristics of WPG single electron transistors (SETs) at $5-20 \mathrm{~K}$. The effect depended on the THz electric field polarization. The observed behavior was explained by photon assisted tunneling based on Tien-Gordon theory.

1 Introduction Solid-state devices operating in the so-called "THz gap" region of electromagnetic waves are highly demanded for possible applications in advanced information technology (IT), biochemistry, nanotechnology and so on. Semiconductor quantum dots have high potentials for solidstate $\mathrm{THz}$ devices, since their energy scales such as subband spacing fall in a few $\mathrm{meV}$ range, corresponding to $\mathrm{THz}$ frequencies.

This paper investigates $\mathrm{THz}$ response of Schottky wrap gate (WPG)-controlled semiconductor quantum dots in view of application to solid-state $\mathrm{THz}$ devices.

The WPG structure and WPG-controlled quantum dot are schematically shown in Figs. 1(a) and (b).

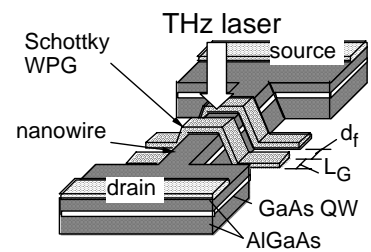

(a)

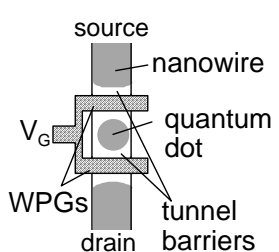

(b)

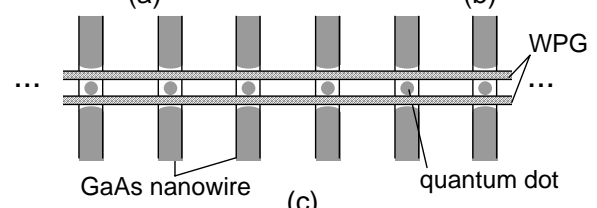

(c)

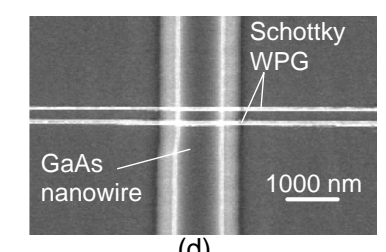

(d)

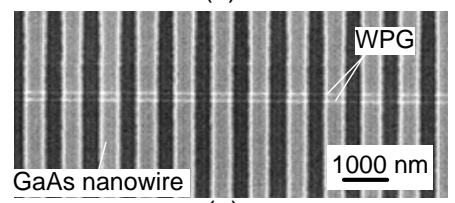

(e)

Fig. 1 (a) Basic structures of WPG SET, (b) and (c) designs of single dot SET and parallel SET integration. (d) and (e) are SEM images of fabricated single dot SET and SET-integrated device, respectively.

Two nanometer-sized Schottky gates, wrapped around a GaAs nanowire with a narrow spacing, form

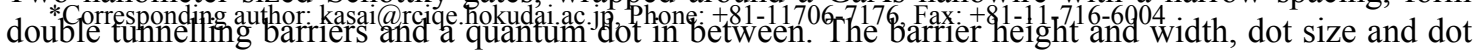
potential are controlled by the gate bias. In the dark, this structure operates as a single electron transistor (SET)[1]. The WPG with simple lateral structure is suitable for planar integration of quantum devices, as 
has been demonstrated recently by a novel hexagonal BDD quantum circuits [2]. In this study, THz response of single dot devices shown in Fig. 1(b) and an integrated device having 50 SETs in parallel shown in Fig. 1(c) were investigated.

2 Experimental GaAs nanowires, having wire widths of a few hundred nm, were fabricated on $\mathrm{AlGaAs} / \mathrm{GaAs}$ heterostructure wafers by EB lithography and wet chemical etching. $\mathrm{Cr} / \mathrm{Au}$ WPGs with a typical length of $85 \mathrm{~nm}$ were formed by EB lithography, metal deposition and lift-off process. SEM micrographs of completed devices are shown in Figs. 1(d) and (e).

Figure 2 shows the experimental set up. THz response of the WPG devices was measured by irradiating a THz (far infrared) laser beam at normal incidence on the samples using a $\mathrm{CH}_{3} \mathrm{OH}$ laser $(\lambda=118$ $\mu \mathrm{m}, v=2.54 \mathrm{THz})$. The sample was placed in a liquid He cryostat, and the SET drain current, $I_{\mathrm{D}}$, was measured as a function of the gate voltage, $V_{\mathrm{G}}$.

3 Result and Discussion Figure 3 shows measured $I_{\mathrm{D}}-V_{\mathrm{G}}$ characteristics of a single dot device with and without $\mathrm{THz}$ irradiation. In this figure, the nanowire direction was parallel to the electric field polarization of the $\mathrm{THz}$ wave as schematically shown in the figure. In the dark, the sample showed a single clear conductance peak followed by a rapid increase of current. The difference of the drain current, $\Delta I_{\mathrm{D}}$, with and without THz irradiation is also shown in Fig. 3 as a function of $V_{\mathrm{G}}$. Here, $\Delta I_{\mathrm{D}}$ was obtained after extracting the exponentially increasing thermal current components from $I_{\mathrm{D}}$ curve by fitting. With $\mathrm{THz}$ beam irradiation, the height of the first peak of the current decreased, showing a negative peak in $\underline{\Delta I_{\mathrm{D}}}$. At the same time, another positive broad peak appeared at a higher gate voltage in the $\Delta I_{\mathrm{D}}-V_{\underline{G}}$ plot. The height of the new peak in the $\Delta I_{\mathrm{D}}$ plot was about $0.7 \mathrm{nA}$. When the nanowire direction was perpendicular to the polarization of the THz wave, no clear change of the current was seen.

The observed modulation cannot be due to thermally heating. In order to understand the experimental result, a theoretical analysis based on the photon assisted tunneling (PAT) theory $[3,4]$ was attempted. Examples of calculated shape of the surrounding tunneling barrier profile and quantum dot are shown in Figs. 4 (a) and (b), respectively. In the dark, basic transport through such a structure can be

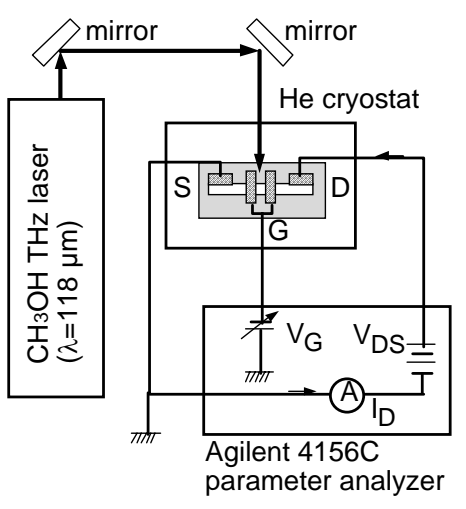

Fig. 2 Measurement set up.

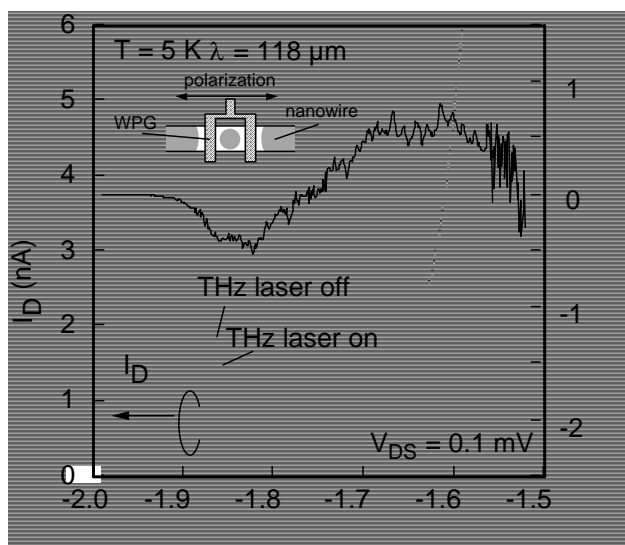

Fig. $3 \mathrm{I}_{\mathrm{D}}-\mathrm{V}_{\mathrm{G}}$ characteristics and $\Delta \mathrm{I}_{\mathrm{D}}-\mathrm{V}_{\mathrm{G}}$ curves.

explained by single electron resonant tunneling,

as we showed previously [1]. The observed rapid exponential increase of current after the single peak is due to thermal transport of electrons over the barriers. Then, the photon assisted tunneling probability, $T_{\mathrm{PAT}}(\mathrm{E})$, through this system, can be calculated by the following formula based on an analysis by TienGordon Hamiltonian [3] on the THz irradiation,

$$
T_{P A T}(E)=\sum_{n=-\infty}^{\infty} \frac{\Gamma_{L} \Gamma_{R} \mid J_{n}\left(q V_{a d} / h v\right)^{2}}{\left(E-E_{0}+n h v\right)^{2}+(\Gamma / 2)^{2}}
$$


where, $\Gamma_{\mathrm{L}}$ and $\Gamma_{\mathrm{R}}$ are the line width of the left and right tunneling barriers, $\Gamma=\Gamma_{\mathrm{L}}+\Gamma_{\mathrm{R}}, E_{0}$ is the quantized state in the dot, $J_{\mathrm{n}}$ is Bessel function, $V_{\mathrm{ac}}$ is amplitude of ac voltage induced by $\mathrm{THz}$ irradiation and $h v$ is $\mathrm{THz}$ photon energy. In this calculation, it was assumed, as a first approximation, that the tunnel barrier profile did not change as the Fermi level was swept with the gate voltage. The energy scale was translated to $V_{\mathrm{G}}$ by $\Delta V_{\mathrm{G}}=\Delta E / \alpha$ with $\alpha=0.05$. The dependence of $\Gamma$ on the quantized energy level was considered and estimated from the calculation of tunneling probability using transfer matrix method. The current was calculated using the $T_{\mathrm{PAT}}(E)$ in the conductance formula[1,5]where the currents of $\mathrm{n}=0, \pm 1$ for the first conductance peak was considered.

An example of the calculated current is shown in Fig. 5(a) for the cases with and without photo assisted tunneling. By THz irradiation, the main peak height decreased and an additional peak appeared at a higher gate voltage. A peak corresponding to $n=-1$ did not appear, since the energy becomes lower than the bottom of the dot potential for a large $\mathrm{THz}$ photon energy of $h v=10.1 \mathrm{meV}$. Theoretical and experimental results are compared in Fig. 5(b) in terms of $\Delta I_{\mathrm{D}}-V_{\mathrm{G}}$ curves. Excellent agreements were

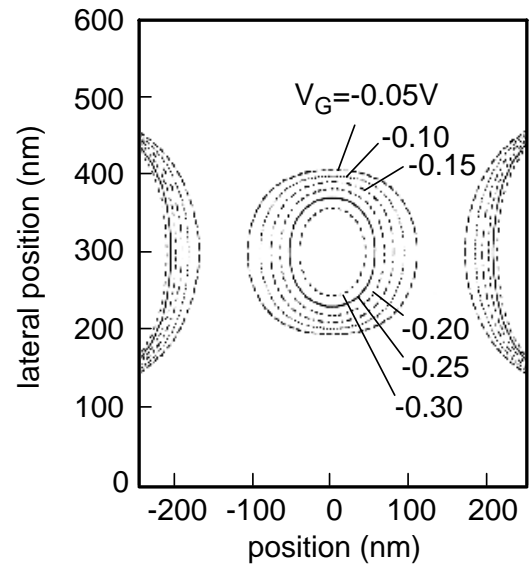

(a)

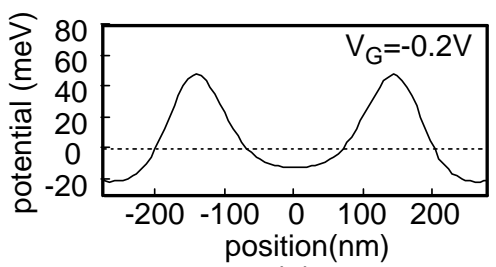

(b)

Fig. 4 Potential simulation on WPG SET: (a) equipotential contour map and (b) potential profile.

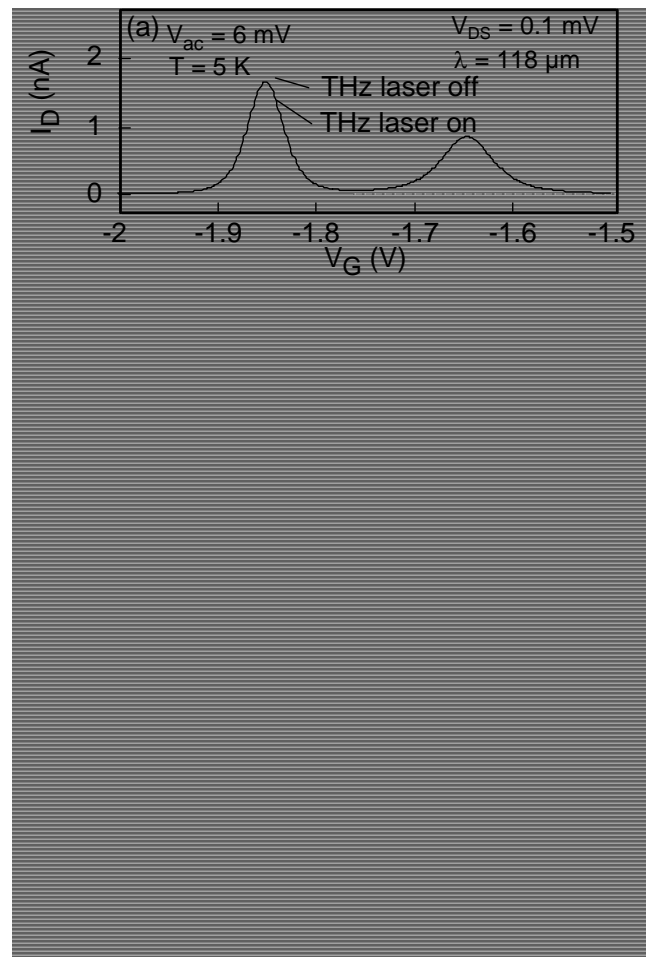

Fig. 5 (a) Example of calculated current peaks by PAT. (b) and (c) are theoretical $\Delta \mathrm{I}_{\mathrm{D}}-\mathrm{V}_{\mathrm{G}}$ curves for various $\mathrm{V}_{\mathrm{ac}}$ and temperatures, respectively.

obtained with the theoretical curve of $V_{\mathrm{ac}}=6$ $\mathrm{mV}$ which seems to be reasonable from the intensity of laser beam. Thus, the experimental characteristics are due to PAT currents. The calculated temperature dependence of $\Delta I_{\mathrm{D}}-V_{\mathrm{G}}$ is also shown in Fig. 5(c). The PAT-induced second peak survives up to $30-50 \mathrm{~K}$, whereas the negative peak quickly disappears and becomes positive, resulting in a single broad peak. This is due to the fact that thermal electrons can tunnel through $n=+1$ PAT channel at elevated temperatures even if the Fermi level lies at $\underline{\mathrm{n}=0 \text { position. }}$ 


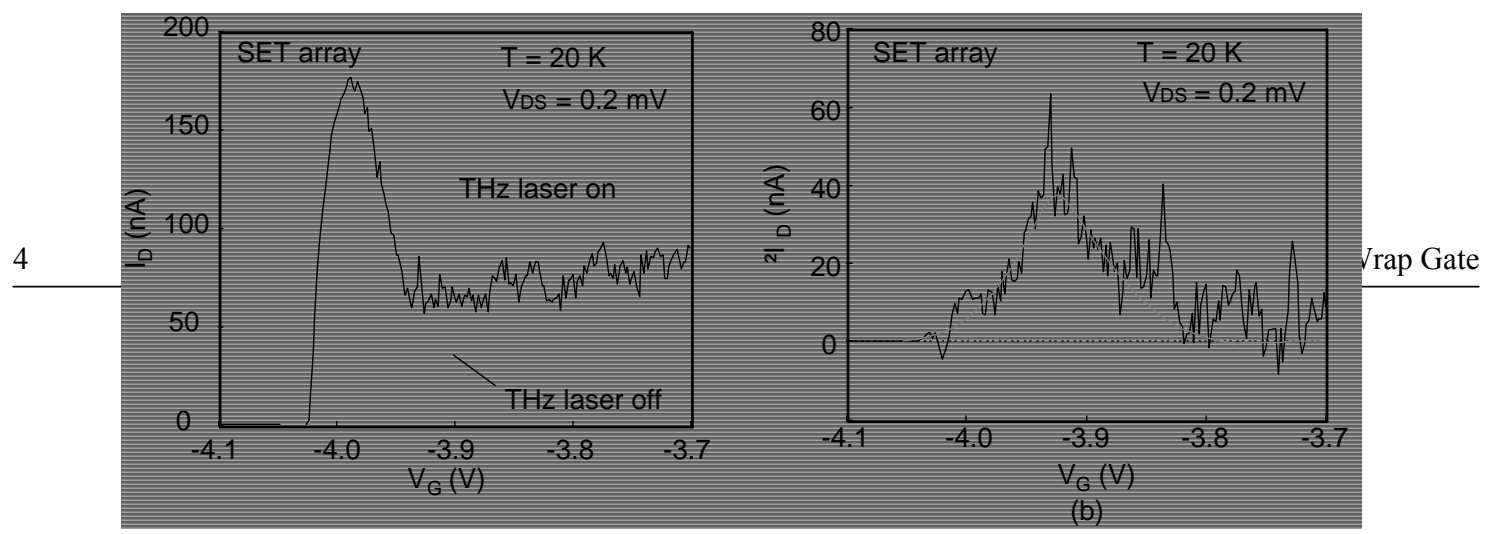

Fig. 6 (a) $\mathrm{I}_{\mathrm{D}}-\mathrm{V}_{\mathrm{G}}$ and (b) $\Delta \mathrm{I}_{\mathrm{D}}-\mathrm{V}_{\mathrm{G}}$ characteristics of device integrating 50 WPGSETs in parallel.

The THz response of the integrated device having 50 SETs in parallel, measured at $20 \mathrm{~K}$, is shown in Figs. 6(a) and (b). The PAT induced a new peak. Since the temperature was relatively high, only a single positive peak was appeared in the $\Delta I_{\mathrm{D}}-V_{\mathrm{G}}$, which is in qualitatively agreement with the result shown in Fig. 5(c). The peak has a large height of $38 \mathrm{nA}$ which roughly corresponds to $0.7 \mathrm{nA} \times 50$. This indicates that it is possible to increase the overall $\mathrm{THz}$ responsivity by forming large array of WPG SETs.

4 Conclusions $\mathrm{THz}$ responses of Schottky wrap gate (WPG)-controlled quantum dots were investigated. Normal incidence irradiation of $\mathrm{THz}$ beam on the single and integrated device surfaces using a $\mathrm{CH}_{3} \mathrm{OH} \mathrm{THz}$ laser $(v=2.54 \mathrm{THz})$ changed conductance behavior and produced an additional conductance peak at $5-20 \mathrm{~K}$. Conductance change depended on the polarization of the beam, indicating the effect is induced by the $\mathrm{THz}$ electric field. The observed characteristics were explained by photon assisted tunnelling. As compared with the quantum well infrared photodetectors (QWIPs), the present device allows normal incidence of $\mathrm{THz}$ beams as well as high-density planar integration. The responsivity of the integrated device was high, being $0.3 \mathrm{~A} / \mathrm{W}$ at $20 \mathrm{~K}$. Thus, it is promising for solid state $\mathrm{THz}$ detectors by enhancing the operation temperature with reducing device feature sizes.

\section{References}

[1] S. Kasai and H. Hasegawa, Proc. of the 25th Int. Conf. on the Physics of Semiconductors, Part II (Springer, Berline, 2001) pp.1813-1814.

[2] S. Kasai and H. Hasegawa, IEEE Electron Device Lett. 23, 446 (2002).

[3] P. K. Tien and J. P. Gordon, Phys. Rev. 129, 647 (1963).

[4] L. P. Kouwenhoven, S. Jauhar, J. Orenstein, P. L. McEuen, Y. Nagamune, J. Motohisa and H. Sakaki, Phys. Rev. Lett. 73, 3443 (1994).

[5] C. W. J. Beenakker, Phys. Rev. B 44, 1646 (1991).

\section{Figure captions}

Fig. 1 (a) Basic structures of WPG SET, (b) and (c) designs of single dot SET and parallel SET integration. (d) and (e) are SEM images of fabricated single dot SET and SET-integrated device, respectively.

Fig. 2 Measurement set up.

Fig. $3 \mathrm{I}_{\mathrm{D}}-\mathrm{V}_{\mathrm{G}}$ characteristics and $\Delta \mathrm{I}_{\mathrm{D}}-\mathrm{V}_{\mathrm{G}}$ curves.

Fig. 4 Potential simulation on WPG SET: (a) equipotential contour map and (b) potential profile.

Fig. 5 (a) Example of calculated current peaks by PAT. (b) and (c) are theoretical $\Delta \mathrm{I}_{\mathrm{D}}-\mathrm{V}_{\mathrm{G}}$ curves for various $\mathrm{V}_{\mathrm{ac}}$ and temperatures, respectively.

Fig. 6 (a) $\mathrm{I}_{\mathrm{D}}-\mathrm{V}_{\mathrm{G}}$ and (b) $\Delta \mathrm{I}_{\mathrm{D}}-\mathrm{V}_{\mathrm{G}}$ characteristics of device integrating 50 WPGSETs in parallel. 